\title{
Numerical heat transfer in a rectangular channel with mounted obstacle
}

\author{
Salim Gareh \\ Department of Mechanical Engineering, University Mohamed Khider, Biskra 07000, Algeria \\ E-mail address: garehsalim@gmail.com
}

\begin{abstract}
The fully-incompressible, viscous and stationary Navier-Stokes equations are solved for the laminar flow over an obstacle placed on the lower of a channel. The Reynolds number is varied from 100 to 400 . In all cases studied the flow field proves to be steady. Several distinct flow features are identified: a horseshoe vortex system, inward bending flow at the side walls of the obstacle, a horizontal vortex at the downstream lower-half of the obstacle and a downstream wake containing two counter-rotating vortices. The shape and size of these flow features are mainly dominated by the Reynolds number. For higher Reynolds numbers, both the horseshoe vortex and the wake region extend over a significantly larger area. The correlation of the position of the separation and attachment point with the Reynolds number has been calculated. A detailed analysis is carried out to investigate flow pattern and Nusselt number.
\end{abstract}

Keywords: laminar flow; obstacle; finite volume simulation; convective heat transfer

\section{INTRODUCTION}

Flow separation in internal flows caused by the presence of a wall-mounted obstacle is of particular relevance to numerous practical engineering applications, due to the associated influence of the pressure loss, heat and mass transfer. Computational research of flow over two-dimensional obstacle mainly focuses on high Reynolds numbers, e.g. [1-7]. For gaseous fluids these studies are typically applicable for large scale objects in low-speed (incompressible) flow. Bilen and Yapici [1] carried out experimental investigations on the effect of orientation angle and geometrical position of wall mounted rectangular blocks. Their results indicated that the most efficient parameters were the Reynolds number and orientation angle. The maximum heat transfer rate was obtained at $450{ }^{\circ} \mathrm{C}$ orientation angle value. Ghia and Davis [2] applied conformal transformations to present solutions to the flow past a semiinfinite obstacle. Young and Vafai [3] investigated the forced convective heat transfer of individual and array of multiple two-dimensional obstacles for a Reynolds number ranging from 800 to 1300. The effect of a change in the channel height and input heat power was investigated and an empirical correlation established. In another study, Wang and Vafai [4] studied the mixed convection and pressure losses in a channel with discrete flush-mounted and protruding heat sources. In the same work, the effect of obstacle geometry and flow rate was considered. An empirical correlation for both pressure drop and Nusselt number was presented. Gareh [5] studied a laminar flow with an obstacle placed on the lower walls. He found that whenever Reynold increases the area tourbillions increases. 
The present work represents a two-dimensional numerical investigation of forced laminar convection in a rectangular channel containing obstacle placed in lower wall.

\section{MATHEMATICAL FORMULATION}

Two-dimensional flow around a surface mounted obstacle can be described by means of the classical continuity, momentum and convection diffusion equations.

To achieve the calculations, these equations and the corresponding boundary conditions are made dimensionless. The characteristic scales

At the inlet section, $\quad \mathrm{p}_{0}=\rho \mathrm{u}_{0}^{2}$

The non-dimensional number.

$$
\begin{array}{r}
R e=\frac{\rho u_{0}}{\mu} \\
\operatorname{Pr}=\frac{v}{\alpha}
\end{array}
$$

and the non-dimensional quantities

$$
\mathrm{u}^{*}=\mathrm{u} / \mathrm{u}_{0}, \mathrm{v}^{*}=\mathrm{v} / \mathrm{v}_{0}, \mathrm{p}^{*}=\mathrm{p} / \rho^{*} \mathrm{u}_{0}^{2}
$$

Are used for that purpose. Therefore, the governing equations in non-dimensional form can be written as:

Continuity:

$$
\nabla * \cdot \mathrm{V}^{*}=\mathrm{O}
$$

X-momentum:

$$
\left(U^{*} \frac{\partial U^{*}}{\partial X^{*}}+V^{*} \frac{\partial U^{*}}{\partial Y^{*}}\right)=-\frac{\partial P^{*}}{\partial X^{*}}+\left(\frac{1}{\operatorname{Re}}\right)\left(\frac{\partial^{2} U^{*}}{\partial X^{* 2}}+\frac{\partial^{2} U^{*}}{\partial Y^{* 2}}\right)
$$

Y-momentum:

$$
\left(U^{*} \frac{\partial V^{*}}{\partial X^{*}}+V^{*} \frac{\partial V^{*}}{\partial Y^{*}}\right)=-\frac{\partial P^{*}}{\partial Y^{*}}+\left(\frac{1}{\mathrm{Re} .}\right)\left(\frac{\partial^{2} V^{*}}{\partial X^{* 2}}+\frac{\partial^{2} V^{*}}{\partial Y^{* 2}}\right)
$$

Energy:

$$
\left(U * \frac{\partial T^{*}}{\partial X^{*}}+V^{*} \frac{\partial T^{*}}{\partial Y^{*}}\right)=\left(\frac{1}{\operatorname{Pr}}\right)\left(\frac{\partial^{2} T^{*}}{\partial X^{* 2}}+\frac{\partial^{2} T^{*}}{\partial Y^{2}}\right)
$$


The applied boundary conditions expressed in the dimensionless form are:

Inflow

$\mathrm{u}=1, \mathrm{v}=0, \mathrm{~T}=0$

Non-slip wall

$\mathrm{y}=0: \mathrm{u}=\mathrm{v}=0$

$\mathrm{T}=0$

$\mathrm{y}=1: \mathrm{u}=\mathrm{v}=0$

$\mathrm{T}=0$

Obstacle

$\mathrm{u}=\mathrm{v}=0, \mathrm{~T}=1$

Outflow

$\partial \mathrm{u} / \partial \mathrm{x}=0, \quad \partial \mathrm{v} / \partial \mathrm{x}=0, \quad \partial \mathrm{T} / \partial \mathrm{x}=0, \quad \partial \mathrm{p} / \partial \mathrm{x}=0$

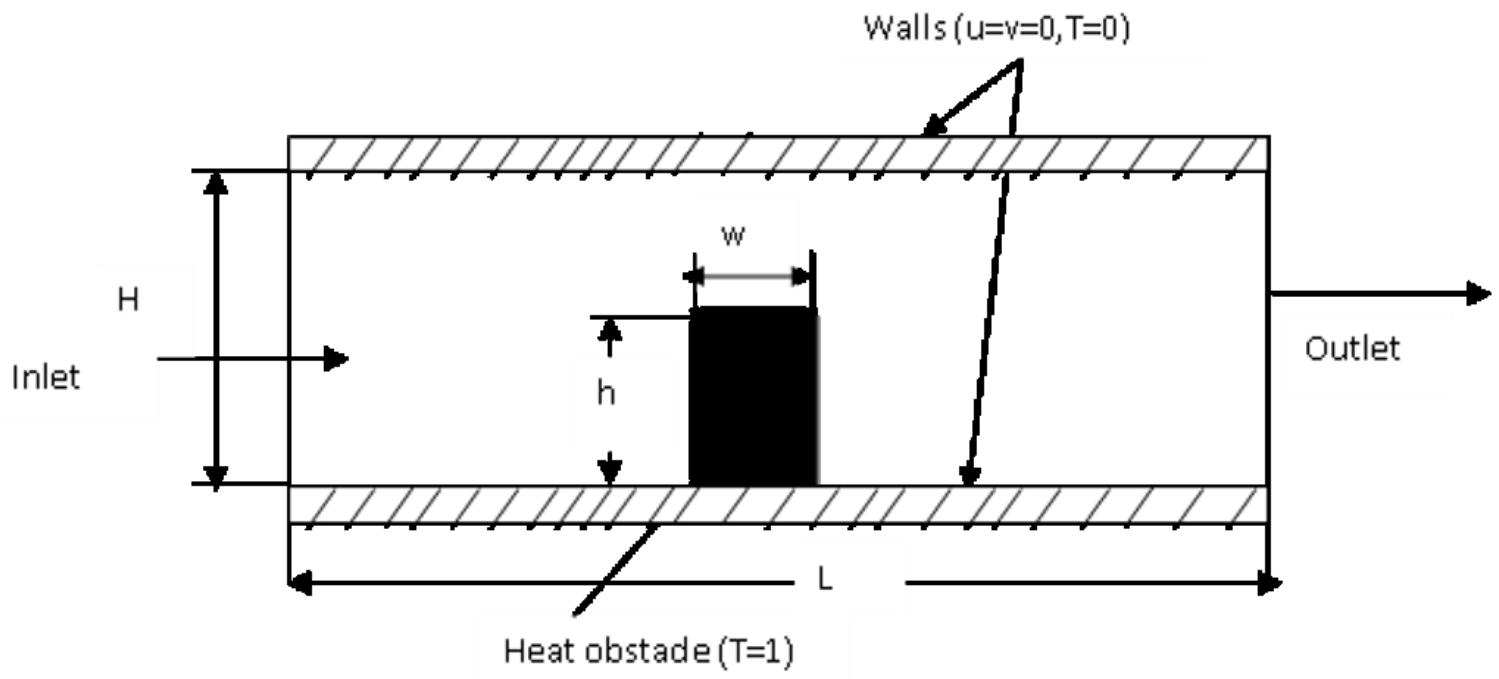

Figure 1. Computational domain.

\section{NUMERICAL SOLUTION}

In the numerical solution of the Navier-Stokes and energy equations, Eqs.(1)-(4), obtained by integrating over an element cell volume. The staggered type of control volume for the x- and y-velocity components was used, while the other variables of interest were computed at the grid nodes. The discretized forms of the governing equations were numerically solved by the SIMPLE algorithm of Patankar [8-10].

Numerical solutions were obtained iteratively by the TDMA method. Numerical calculations were performed by writing a computer program in MATLAB 7.1. The convergence criterion was assumed to have been achieved when the values of residual terms in the momentum and energy equations did not exceed $10^{-3}$. As the result of grid tests for obtaining the grid-independent solution, an optimum grid of $41 \mathrm{x} 41$ is determined in the $\mathrm{x}$ 
and y directions, respectively. Another hand, we are based in this paper on the Code of the Gareh [10].

\section{RESULTS AND DISCUSSION}

\section{1. Dynamical study}

The dimensionless parameters to be considered and which characterize the flow field and heat transfer are as follows: The Reynolds number based on channel height is taken equal to $100,200,300$ and 400 , the obstacle dimensions $(\mathrm{h}, \mathrm{w})$ and the obstacle stream wise spacing (L) are taken as $\mathrm{h}=0.5, \mathrm{w}=\mathrm{h} / 2$ and $\mathrm{L}=1$.

Local Nusselt number defined by the local temperature gradient at the wall:

$$
N u x=\left(\frac{\partial T}{\partial y}\right)_{x=0, h}
$$

The average Nusselt number is defined by

$$
N u=\int_{0}^{l} N u x d x=\int_{0}^{l}\left(\frac{\partial T}{\partial y}\right)_{x=0, h} d x
$$

The dimensionless parameters that must be specified and which characterize the flow field and heat transfer are the Reynolds number the obstacles dimensions, $\mathrm{h}$, wands baseline configuration $(\mathrm{h}=\mathrm{w}=0.5)$. For the effect of obstacles dimensions. By considering the evolution of the flow and thermal fields in the range of Reynolds number from 100 to 400, it was found that the transition from steady to unsteady flow occurs at low Reynolds number when compared to the channel with no obstacle on the lower wall.

The vortex in inter-obstacle groove is seen to be the most affected. The fluid flow then interacts more with the obstacle faces as illustrated by Figure 1a which shows the streamlines for $\mathrm{Re}=100$ and for a baseline configuration $\mathrm{h}=\mathrm{w}=0.5$. It is observed that the flow tends to enter in-between obstacle cavities.

For higher values of the Reynolds number (Figure $1 b$ and $c$ ), the flow becomes unsteady periodic and the length of the recirculation zone seems to get reduced when compared with the configuration without obstacle on the upper wall. Besides, the recirculation zone behind the last obstacle is more pronounced. In the transition from steady to unsteady periodic flows, a wave is induced by vortex shedding behind the last obstacle. This wave results in a change of the reticulating zone behind the latter. 

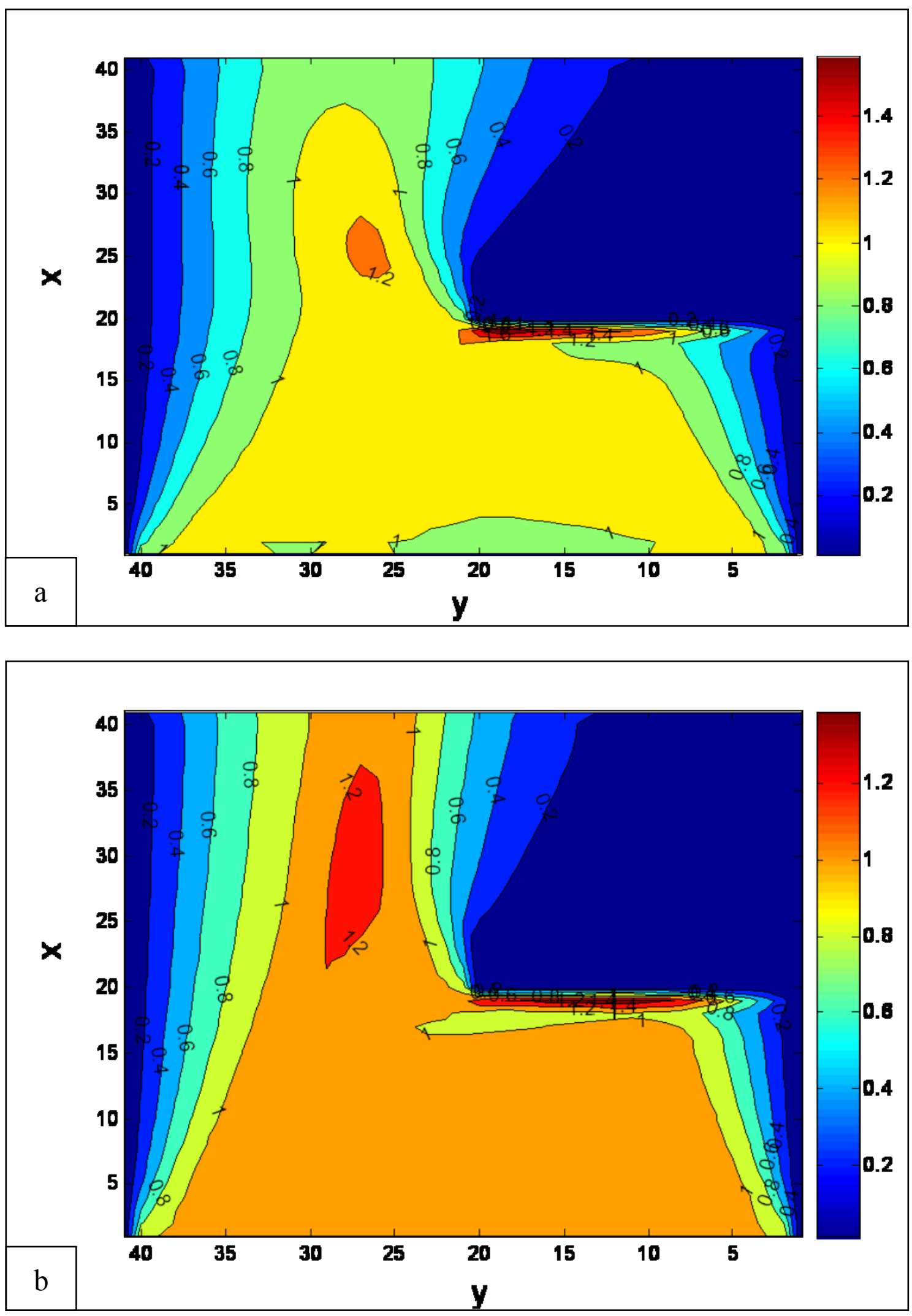


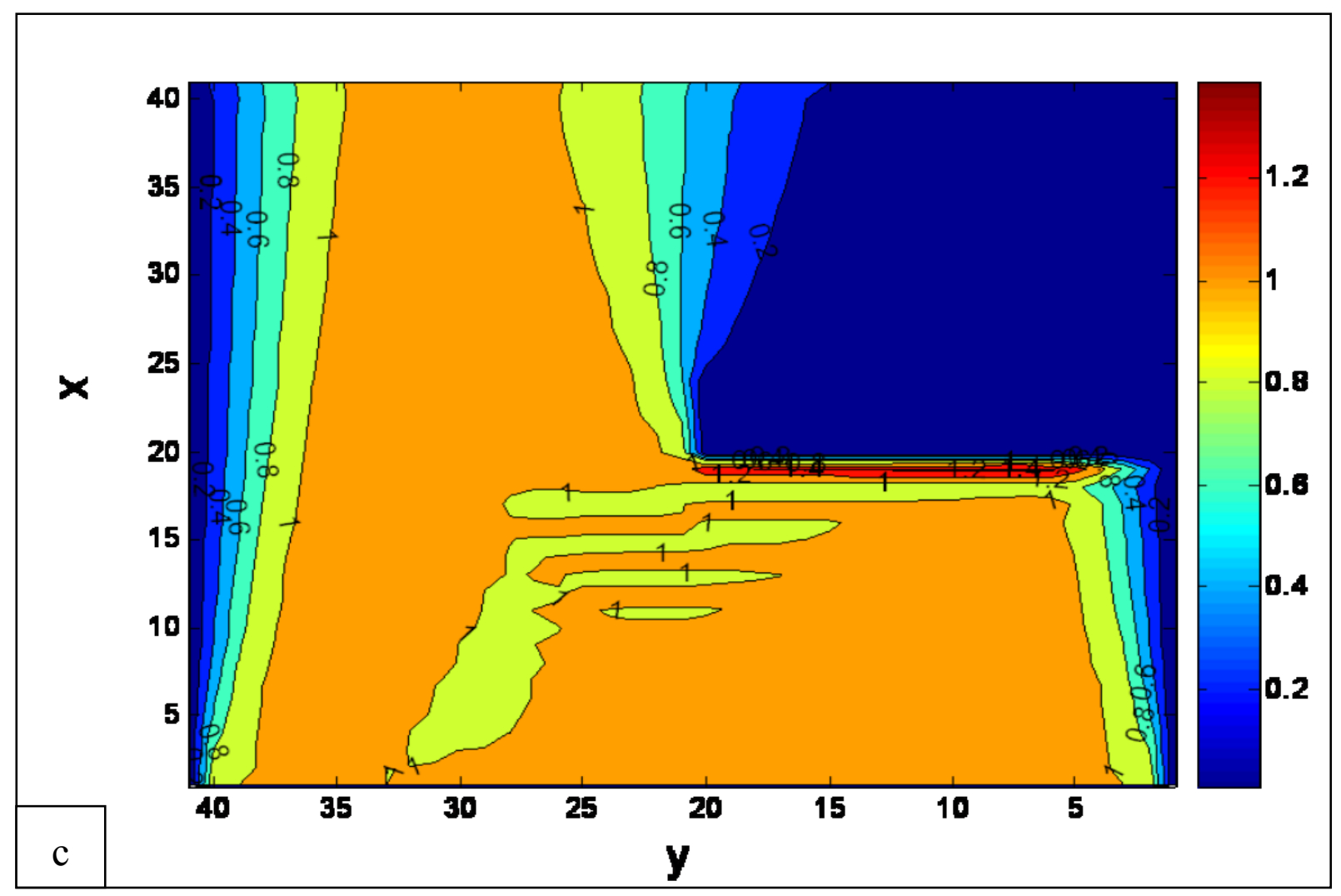

Figure 1. Streamlines for three values of Reynolds number: (a) $\operatorname{Re}=100$, (b) $\operatorname{Re}=200$, (c) $\operatorname{Re}=400$.

\section{2. Thermal study}

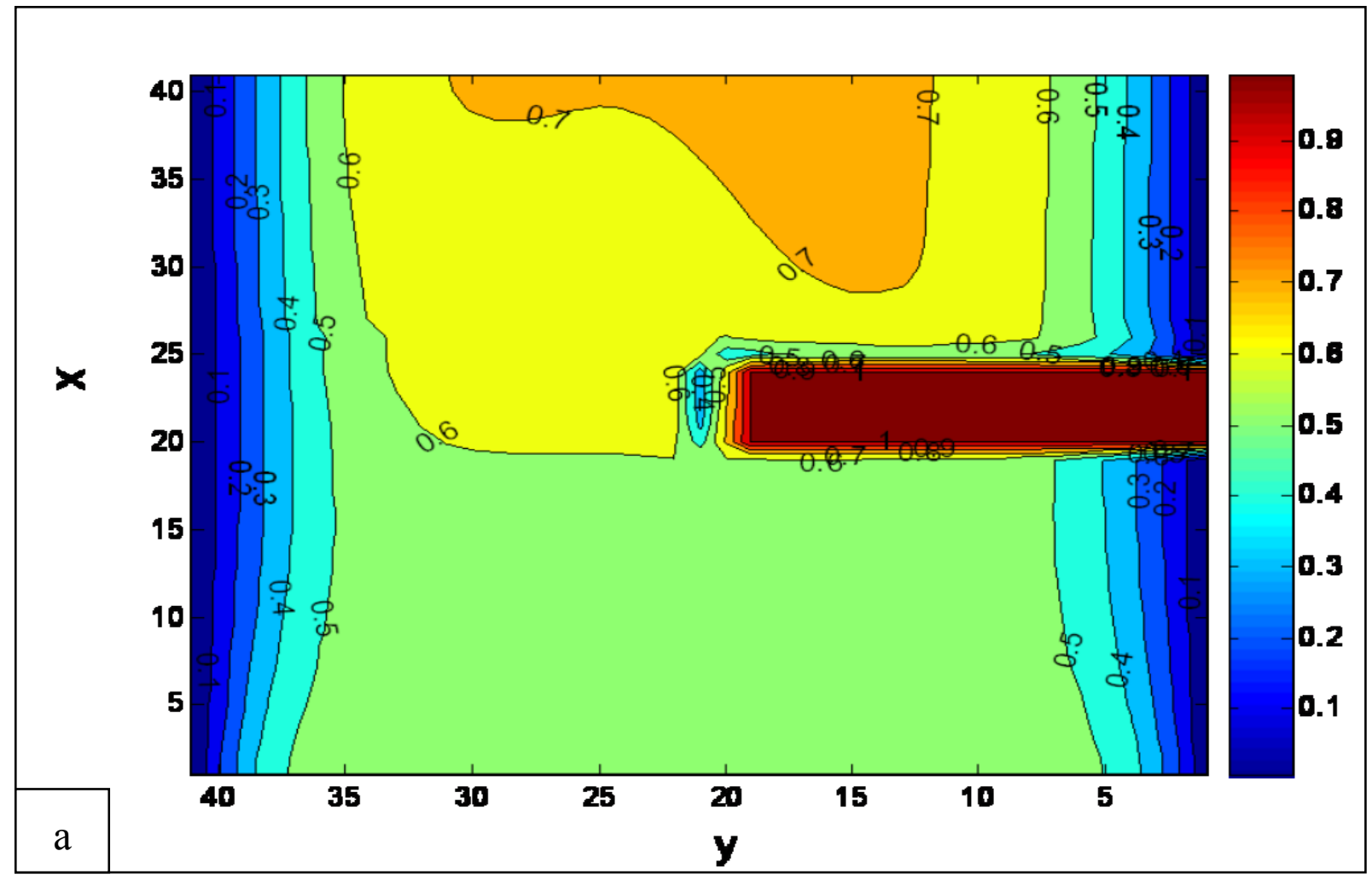



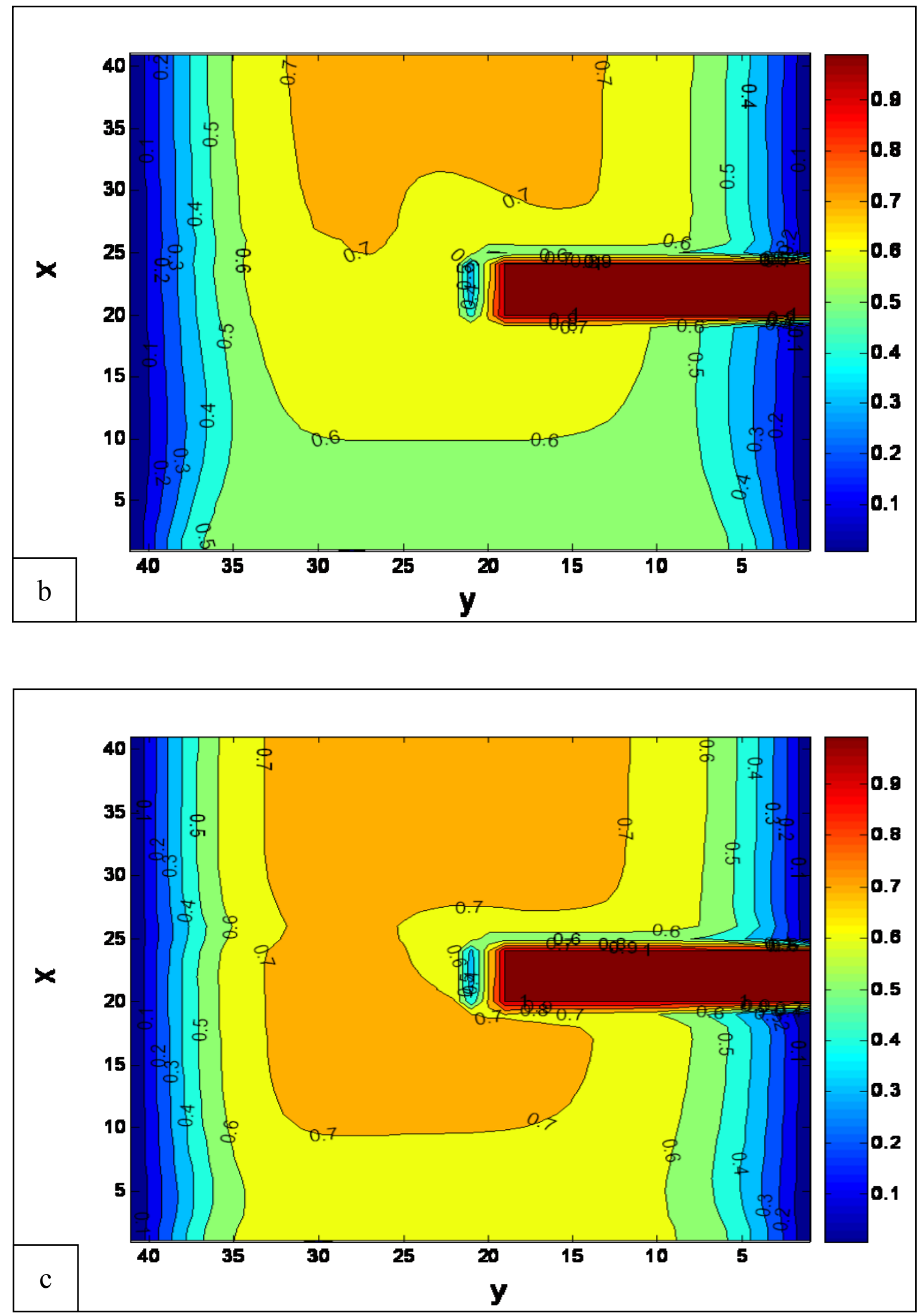

Figure 2. Isotherms contours at steady state for different Reynolds number: (a) $\operatorname{Re}=100$, (b) $\operatorname{Re}=$ 200, (c) $\operatorname{Re}=400$. 
The representation of the isotherm contours obtained for different values of the Reynolds number is shown in Figure 2. It is observed that the isotherm lines are denser close to the upstream and the top faces of the obstacles. Furthermore, the isotherm contours near the downstream face of the obstacles are denser than those obtained in channels without obstacle on the upper wall. It is also observed that when the Reynolds number increases, a vortex appears inside the inter-obstacle cavities and the isotherm contours become thoroughly denser especially near the faces. This yields to the removal of higher quantities of energy from both the right and the left obstacle faces.

Figure 3. Represents the Nusselt number obtained of the obstacle but for values of the Reynolds number equal to $100,200,300$ and 400 . As expected, it can be clearly observed that values of the Nusselt number become higher with increasing values in the Reynolds number. The Nusselt numbers curve for the left face of the obstacle present a local maximum in the face distance. It is believed that such a phenomenon is the result of flow redirection toward the opposite wall by the obstacle. The value of the Nusselt number at the right face of the obstacle is nearly constant and has always a positive value.

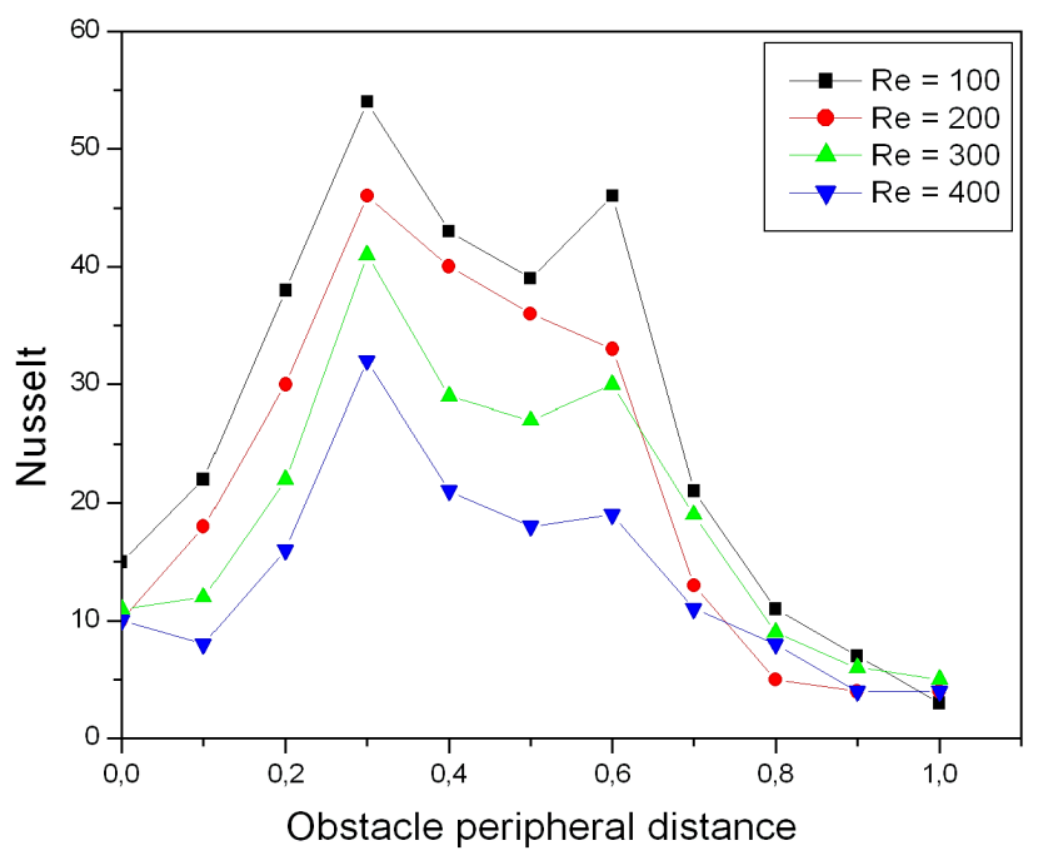

Figure 3. Evolution of the Nusselt number along obstacle peripheral distance for different Reynolds number $\mathrm{Re}=100,200,300$ and 400.

\section{CONCLUSION}

The study first showed that when an obstacle is added on the lower wall of the channel, the transition from steady to unsteady flow is obtained at lower values of the Reynolds number. The various isotherms and Nusselt number curves were presented. The results obtained showed that as the value of the Reynolds number was increased, the heat removed from the obstacles increased sensibly with a maximum heat removal around the obstacle corners. However, vortex shedding generated by the obstacle on the lower wall can 
additionally enhance heat transfer along the obstacle surfaces. The wavy flow significantively changes the reticulating zone behind the last obstacle.

\section{References}

[1] K. Bilen, S. Yapici, Heat and Mass Transfer 38 (2001) 649-655.

[2] T.J. Young, K. Vafai, Journal Heat Transfer 121 (1999) 34-42.

[3] Y. Wang, K. Vafai, Experimental Heat Transfer 12 (1999) 1-16.

[4] S. Gareh, Journal of Chemistry and Materials Research 1 (2014) 34-38.

[5] D. Greenspan, Journal Engineering Mathematic (1969) 21-28.

[6] S.H. Kim, N.K. Anand, Numerical Heat Transfer Part A 38 (2001) 1-21.

[7] S.V. Patankar, Numerical Heat transfer and Fluid Flow, Hemisphere Publishing Corporation, Taylor \& Francis Group, New York, USA, 1980.

[8] H.K. Versteeg, W. Malaskera, An introduction of computational fluid dynamics, Longman Group Ltd England 1995.

[9] S. Gareh, Journal of Chemistry and Materials Research 1 (2014) 7-11. 